\title{
Brian Holloway Appointed MRS/OSA Congressional Fellow for 1997-98
}

Brian Holloway of Stanford University is the recent recipient of the 1997-1998 MRS/OSA Congressional Science and Engineering Fellowship. As a precursor to a career involving public service and technology transfer, he will work for one year in a Washington congressional office. "I hope to learn how scientific policy is formulated in order to someday focus on basic scientific-research funding," said Holloway.

The goal of the fellowship is to promote communication between Congress and the science and engineering community. Fellows serve with individual members of Congress, committees, or support agencies such as the Congressional Budget Office and the Congressional Research Service. According to MRS Public Affairs Committee Chair Julia Phillips, the program has greatly benefitted the Society. "The Fellows are extremely energetic and productive. For example Kelly Kirkpatrick, the first MRS/OSA Fellow, has influenced legislation such as the recently proposed tax on waived graduate-student tuition." At press time for this issue of MRS Bulletin, the bill had passed through Congress, retaining section $117 \mathrm{~d}$ of the Internal Revenue Code. This segment of law ensures graduatestudents' continued exemption from tax responsibility on the value of tuition

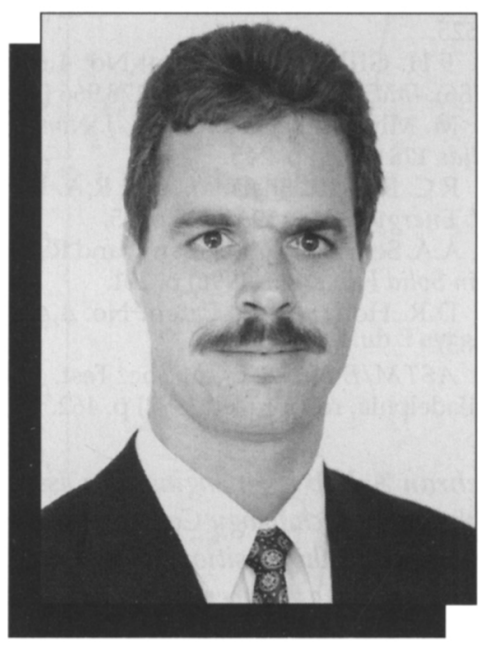

Brian Holloway

waivers. "Kelly has also provided MRS members with valuable insight into the legislative process through her efforts to improve the MRS Public Affairs homepage as well as her efforts on pubic-affairs issues," said Phillips.

Holloway will start with a two-week orientation at the American Association for the Advancement of Science, which coordinates the fellowship. "I hope to bring my youthful enthusiasm and my wide variety of scientific interests to the position," he said. Having recently completed his $\mathrm{PhD}$ degree in mechanical engineering with a minor in materials science and engineering, Holloway is investigating a congressional office where he feels his services would be most helpful. He plans to make a decision in late September or early October. Kirkpatrick served Senator Joseph I. Lieberman (D-CT) during her fellowship (see MRS Bulletin, Vol. XX, No. 8, p. 64). The 1996-1997 Fellow, Michal Freedhoff, worked for U.S. Congressional Representative Edward J. Markey (D-MA). (For more information on Freedhoff's fellowship, see MRS Bulletin, Vol. 21, No. 10, p. 74; and MRS Bulletin, Vol. 21, No. 11, p. 74.)

"I want to thank MRS and OSA for providing me with this once-in-a-lifetime opportunity," Holloway said.

Holloway's dissertation work involved studying the chemical bonding present in carbon nitride thin films in order to determine the chemical processes occurring during film deposition. He received a BS degree in mechanical engineering from the University of Florida. During his undergraduate years, Holloway coowned and operated a commercial fishing company.

\section{INTERNATIONAL UNION OF MATERIALS RESEARCH SOCIETIES}

\section{IUMRS-ICEM-98 Issues Call for Papers}

The Fourth International Conference on Electronic Materials (IUMRS-ICEM-98), chaired by Soon Ja Park (Seoul National University) with Min Che Chon (Chon, International) as honorary chair, will be held August 24-27, 1998, in Cheju, Korea. The conference, organized by Materials Research Society of Korea (MRS-K) under the auspices of the International Union of Materials Research Societies (IUMRS), is a continuation in the series of meetings held in Strasbourg, France (1992); Hsinchu, Taiwan (1994); and Boston, USA (1996). Prospective authors should submit five copies of a 1,000 word summary to Professor Shinhoo Kang, The Secretariat of IUMRS-ICEM-98, Materials Research Society of Korea, School of Materials Science and Engineering, Seoul National University, Seoul 151-742, Korea; 82-2-8807167; fax 82-2-884-1413; e-mail icem98@ plaza.snu.ac.kr. The deadline is February 28, 1998.

ICEM has provided a forum focused on areas of preparation, characterization, and application of electronic materials. The meeting will consist of ten symposia, which emphasize the latest developments in research areas of various electronic materials: Si-based Processing; Compound Semiconductors; Electro-Ceramics and Sensors; Display Materials; Magnetics; Ferroelectric Thin Films; Electronic Packaging; Surface, Interfaces, and Nanotechnology; Polymers for Electronics; and Energy Conversion Storage Materials.

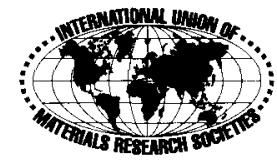

News about IUMRS conferences and other activities should be sent to:

MRS Bulletin, Materials Research Society 506 Keystone Drive, Warrendale, PA 15086-7573 USA

E-mail: bulletin@mrs.org 


\section{San Francisco Marriott Hotel, Salon 8 and Salon 9, Tuesday-Thursday, April 14-16, 1998}

\section{SPRING MEETING}

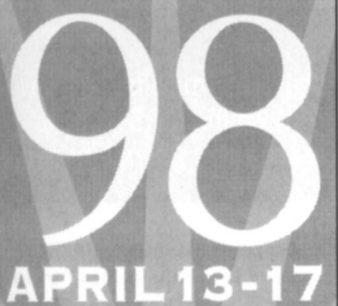

\section{SAN FRANCISCO}

CALIFORNIA

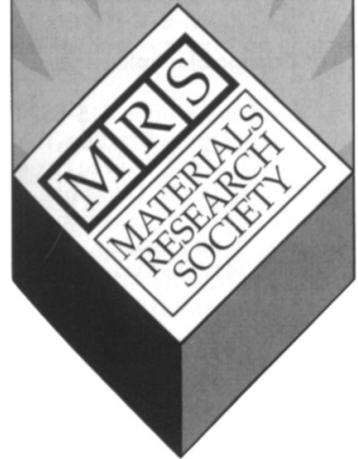

MRS encourages attendees to visit the exhibit by:

\section{- Programming both moming and aftemoon complimentary coffee breaks in the Exhibit Hall every day.}

\section{- Offering a deli-style lunch for purchase in the Exhibit Hall every day from noon to 1:30 p.m.}

\section{- Hosting a complimentary reception in the Exhibit Hall on Tuesday evening from 5:00 p.m. to 6:30 p.m.}

The MRS Exhibit, held in conjunction with the 1998 MRS Spring Meeting, will feature over 120 international exhibitors who will display a full spectrum of equipment, instrumentation, products, software, publications and services for materials research. The exhibit will closely parallel the nature of the technical symposia and, as always, the program has been arranged to allow meeting participants ample opportunity to visit the exhibit.

Exhibit Hours (tentative)

Tuesday, April 14 . . . . . 11:30 a.m. - 6:30 p.m.*

Wednesday, April 15 . . . . 9:30 a.m. - 6:00 p.m.

Thursday, April 16 . . . . 9:30 a.m. - 1:30 p.m.

${ }^{\star}$ Complimentary Reception from 5:00 p.m. - 6:30 p.m.

\section{List of 1997 Spring Exhibitors}

Booth Space is limited...contact MRS for your application today!

\section{A \& N Corporation}

\section{ABB Extrel}

Academic Press, Inc.

Accurel Systems International Corporation

Aetrium, Inc.

AIXTRON, Inc.

AJA International, Inc.

Aldrich Chemical

Company, Inc.

American Institute of Physics, Inc.

APD Cryogenics, Inc.

ASTeX/Applied Science and Technology, Inc.

Bede Scientific Incorporated

Blake Industries, Inc.

Burleigh Instruments, Inc.

Ceramaseal

$\mathrm{CHA}$ Industries

Chemat Technology, Inc.

Commonwealth Scientific Corporation

Creekside Technologies

Cryomech, Inc.

DCA Instruments, Inc.

Denton Vacuum, Inc.

Digital Instruments, Inc.

Duniway Stockroom

Eagle-Picher Industries

EDAX International

Elsevier Science

EMCORE Corporation

EMiSPEC Systems, Inc.

EPI/MBE Products Group

Epion/PVD Products

ERG Materials \&

Aerospace Corporation

ESCETE B.V.

ESM Software

Charles Evans \&

Associates
FEI Company, Components Group

E. A. Fischione Instruments, Inc.

Fujikin of America, Inc.

Gatan, Inc.

General Vacuum, Inc.

Heraeus Amersil, Inc.

High Voltage Engineering

Huntington Mechanical Laboratories, Inc.

Hysitron, Inc.

IBM Analytical and Test Services

Implant Sciences

Corporation

Inel, Inc.

InnoVac Corporation

Instruments SA, Inc.

Insulator Seal Inc.

Ion Tech, Inc.

Janis Research Company

JEOL USA, Inc.

k-Space Associates, Inc. Keithley Instruments, Inc.

Kevex Instruments, Inc.

Kevex X-Ray Inc.

Kluwer Academic Publishers

Lake Shore Cryotronics

Lambda Physik, Inc.

Lambda Technologies

Lasertec U.S.A., Inc.

Kurt J. Lesker Company

LUXTRON Corporation

Magnet Sales \& Manufacturing

MARCH Instruments, Inc.

Materials Analysis Group/ Philips Semiconductors

Materials Technologies International (MTI)
MDC Vacuum Products Corporation

Melles Griot, Inc.

Micrion Corporation

Micro Photonics, Inc.

MMR Technologies, Inc.

Molecular Simulations, Inc.

MVSystems, Inc.

Nano Instruments, Inc.

Nanofilm Technologie $\mathrm{GmbH}$

National Electrostatics Corporation

Neocera, Inc.

New Focus, Inc.

Nicolet Instrument Corporation

Nikko Hitech International

Noranda Advanced Materials

Nor-Cal Products, Inc.

Omicron Associates

Oxford Applied Research

Oxford Instruments Scientific Research Division

Park Scientific Instruments

Parke Mathematical Laboratories

Philips Electronic Instruments Company

Physical Electronics, Inc.

Plasma Sciences, Inc.

Plasmatron Coating Systems, Inc.

Princeton Instruments, Inc.

Pure Tech, Inc.

QualiTau, Inc.

Renishaw Inc.

Research and PVD

Materials Corporation

Rigaku/USA, Inc.

$\mathrm{RMC}$
SC Technology/Telemark SensArray Corporation Shiva Technologies, Inc. SKION Corporation

South Bay Technology, Inc.

SPI Supplies/Structure

Probe, Inc.

STAIB Instruments, Inc.

Struers/Logitech Product Group

SURFACE

Surface/Interface, Inc.

SVT Associates, Inc.

TECHNOTRADE

International, Inc.

Tencor Instruments

TexSEM Laboratories

(TSL), Inc.

Thermionics Laboratory

Thomas Swan \& Co., Ltd.

TopoMetrix Corp.

Union Carbide Crystal

Products

Vacuum Atmospheres Co.

Varian Vacuum Products

VCR Group, Inc.

Virginia Semiconductor

Voltaix, Inc.

Yasui Seiki Co. (USA)

For your 1998 MRS Spring Exhibit Application, contact:

Mary E. Kaufold, Manager Advertising \& Exhibits Materials Research Society Telephone: (412) 779-8312

Fax: (412) 779-4397

E-mail: kaufold@mrs.org 

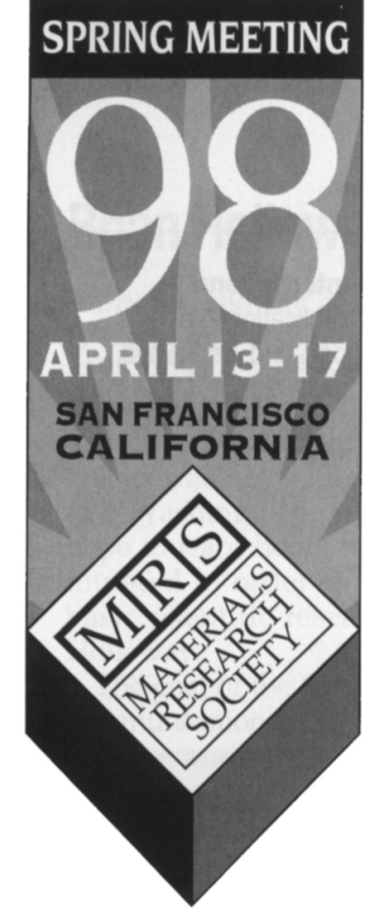

Exhibit: April 14-16

\section{Spring Meeting Chairs}

John A. Emerson Organic Materials Department 1472 MS0958 Sandia National Labs Albuquerque, NM 87185-0958 Phone: 505-845-9747 Fax: $505-844-2894$ jaemers@sandia.gov

Ronald Gibala

Department of Materials Science and Engineering 2026 H.H. Dow Building

Univ. of Michigan 2300 Hayward Street Ann Arbor, MI 48109-2136 Phone: 313-936-0178 Fax: $\quad 313-763-4788$ rgibala@umich.edu

\section{Caroline A. Ross}

Department of Materials Science and Engineering Room 13-4005

Massachusetts Inst. of Technology

77 Massachusetts Avenue Cambridge, MA 02139 Phone: 617-258-0223 Fax: $617-252-1020$ caross@mit.edu

\section{Leo J. Schowalter}

Physics Department and Center for Integrated Electronics Rensselaer Polytechnic Inst. 110 - 8th Street Troy, NY 12180-3590 Phone: $518-276-6435$ Fax: $518-276-6680$ schowalt@unix.cie.rpi.edu

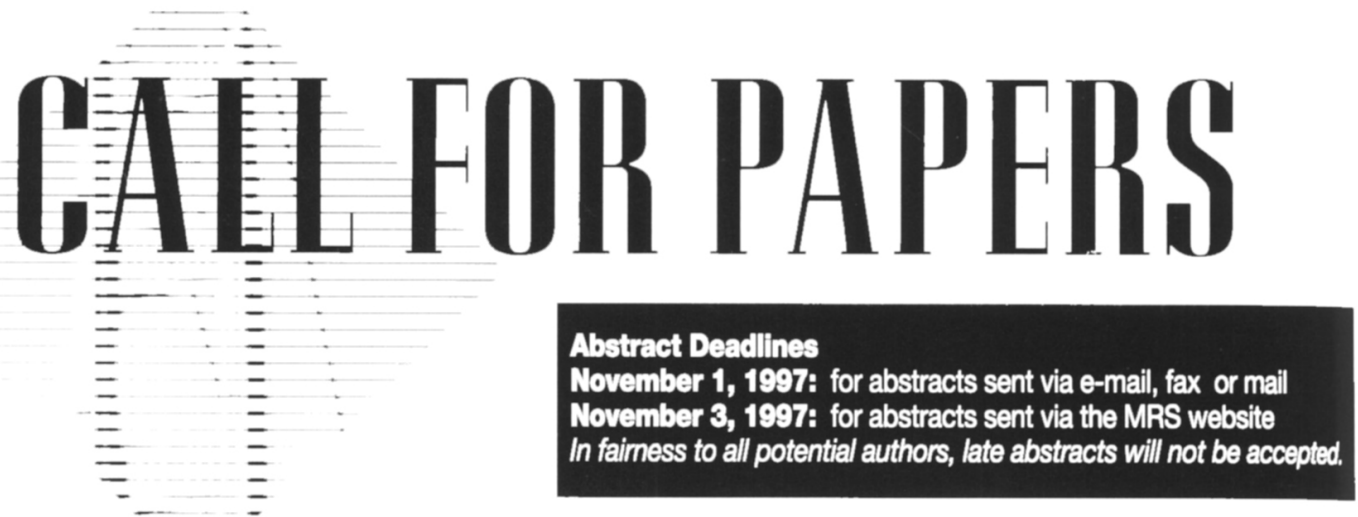

Symposia and Symposium Organizers

A: Amorphous and Microcrystalline Silicon Technology - 1998 Ruud E.I. Schropp, Utrecht Univ., The Netherlands, Phone 31-30-2533170, Fax 31-30-2543165, schropp@fys.ruu.nl; Howard M. Branz, National Renewable Energy Lab, Phone 303 384-6694, Fax 303-384-6531, hbranz@nrel.gov; Michael Hack, dpiX, a Xerox Co., Phone 415-812-4535, Fax 415-493-6349, hack@dpix.com; Isamu Shimizu, Tokyo Inst. of Technology, Japan, Phone 81-45-924-5424, Fax 81-45-921-1089, shimizu@echem.titech.ac.jp; Sigurd Wagner, Princeton Univ., Phone 609-258-4631, Fax 609-258-6279, wagner@princeton.edu Administrative contact: Mary Ann Woolf, Univ. of Utah, Phone 801-581-4246, Fax (same as phone) or -4801; woolf@pclab. physics.utah.edu

\section{B: Flat-Panel-Display Materials and Large-Area Processes} Ted S. Fahlen, Candescent Technologies Corp., Phone 408360-2650, Fax 408-229-0850, tfahlen@candescent.com; Shinji Morozumi, Hosiden and Philips Display Corp., Japan, Phone 81-78-9915791, Fax 81-78-9978652, morozumi@hosiden. co.jp; Gregory Parsons, North Carolina State Univ., Phone 919 515-7553, Fax 919-515-3465, parsons@ncsu.edu; Carl Seager, Sandia National Labs, Phone 505-844-9168, Fax 505-844-1197, chseage@sandia.gov; Chuang-Chuang Tsai, Applied Komatsu Technology, Phone 408-235-6847, Fax 408-563-0685, cc_tsai@amat.com

\section{C: Materials Issues in Vacuum Microelectronics}

Wei Zhu, Lucent Technologies/Bell Labs, Phone 908-582-7659, Fax 908-582-4868, wzhu@bell-labs.com; Lawrence S. Pan, Candescent Technologies Corp., Phone 408-360-2667, Fax 408229-8008, Ipan@candescent.com; Thomas E. Felter, Lawrence Livermore National Lab, Phone 510-422-8012, Fax 510-4244737, felter1@|Inl.gov; Christopher Holland, SRI International, Phone 415-859-6382, Fax 415-859-3090, cholland@unix.sri.com

\section{D: Defect and Impurity Engineered Semiconductors and} Devices II

S. Ashok, The Pennsylvania State Univ., Phone 814-863-4588, Fax 814-863-7967, sxa4@psu.edu; K. Sumino, Nippon Steel Corp. Japan, Phone 81-439-802260, Fax 81-439 802769 , sumino@kikaku.re.nsc.co.jp; J. Chevallier, CNRS, France, Phone 33-1-45075340, Fax 33-1-45075841, chevaler@cnrs-bellevue.fr B.L. Sopori, National Renewable Energy Lab, Phone 303-3846683, Fax 303-384-6684, soporib@tcplink.nrel.gov; W. Goetz Hewlett Packard Co., Phone 408-435-6007, Fax 408-435-6335, werner_goetz@hp.com

\section{E: Low-Dielectric Constant Materials and Appllcations in} Microelectronics II

Chien Chiang, Intel Corp., Phone 408-765-2721, Fax 408-7652061, chien_chiang@ccm.sc.intel.com; Toh-Ming Lu, Rensselaer Polytechnic Inst., Phone 518-276-8379, Fax 518-276-6680, tmlu@unix.cie.rpi.edu; Jeffrey T. Wetzel, Motorola Inc., Phone 512-933-5039, Fax 512-933-5497, rxmn70@aprdlgtr.sps. mot.com; Paul S. Ho, Univ. of Texas, Austin; Phone 512-4718961, Fax 512-471-9674, paulho@mail.utexas.edu

\section{F: Wlde-Bandgap Semiconductors for High Power, High} Frequency and High Temperature

Steve Denbaars, Univ. of California, Santa Barbara, Phone 805893-8511, Fax 805-893-8983, denbaars@engrhub.ucsb.edu; John Palmour, Cree Research, Inc., Phone 919-361-4770, Fax 919-361-2266, john_palmour@cree.com; Michael S. Shur Rensselaer Polytechnic Inst., Phone 518-276-2201 or -6019, Fax 518-276-6261, shur@ecse.rpi.edu, http://nina.ecse.rpi.edu/shur/; Michael Spencer, Howard Univ., Phone 202-806-6618, Fax 202806-6597, spencer@msrce.howard.edu

\section{G: Science and Technology of Organic Electroluminescent} Devices

Homer Antoniadis, Hewlett-Packard Labs, Phone 415-857-2386, Fax 415-857-2379, homer@hpl.hp.com; Donal Bradley, The Univ. of Sheffield, United Kingdom, Phone 44-114-2224575, Fax 44. 114-2728079, d.bradley@sheffield.ac.uk; Ian H. Campbell, Los Alamos National Lab, Phone 505-667-5757, Fax 505-665 4292, campbell@lanl.gov; Junji Kido, Yamagata Univ., Japan, Phone 81-238-263052, Fax 81-238-263412, kid@dipfr.dip.yz. yamagata-u.ac.jp

\section{H: Hydrogen in Semiconductors and Metals}

Norbert H. Nickel, Hahn-Meitner-Institut Berlin, Germany, Phone 49-30-67053301, Fax 49-30-67053333, nickel@hmi.de; Warren B. Jackson, Xerox Palo Alto Research Lab, Phone 415-812-4196, Fax 415-812-4105, wjackson@parc.xerox.com; Robert C. Bowman, Jet Propulsion Lab, Phone 818-354-7941, Fax 818393-4206, robert.c.bowman-jr@jpl.nasa.gov; Robert G. Leisure, Colorado State Univ., Phone 970-491-5370, Fax 970-491-7947, leisure@lamar.colostate.edu

\section{I: Advanced Interconnects and Contact Materlals and} Processes for Future lcs

Shyam P. Murarka, Rensselaer Polytechnic Inst., Phone 518276-2978, Fax 518-276-2990, smurarka@unix.cie.rpi.edu; Mosh Eizenberg. Technion-Israel Inst. of Technology, Israel, Phone/Fax 972-4-8294585, eizen@tx.technion.ac.il; David B. Fraser, Intel Corp., Phone 408-765-2926, Fax 408-653-5157, david_b_frasere $\mathrm{ccm}$.hf.intel.com; Roland Madar, Institut National Polytechnique de Grenoble/ENSPG, France, Phone 33-476-826330, Fax 33-476829394, roland.madar@adminpg.fr; Raymond Tung, Lucent Technologies/Bell Labs, Phone 908-582-6895, Fax 908-582-2300, rtt@clockwise.lucent.com

\section{J: Electronic Packaging Materials Science $X$}

Daniel J. Belton, Intel Corp., Phone 602-554-8963, Fax 602-554 7813, daniel_j_belton@ccm.hf.intel.com; Michael Gaynes, IBM Corp., Phone 607-755-9244, Fax 607-755-9192, mgaynes@ vnet.ibm.com; Raymond Pearson, Lehigh Univ., Phone 610-758 3857, Fax 610-758-4244, rpo2@lehigh.edu; Elizabeth G. Jacobs, Texas Instruments, Phone 972-995-4703, Fax 972-995-2658, zzil@ti.com

\section{K: Materials Reliability in Microelectronics VII}

Thomas Marieb, Intel Corp., Phone 408-765-5255, Fax 408-7652949, thomas_marieb@ccm.sc.intel.com; John Bravman, Stanford Univ., Phone 415-723-3698, Fax 415-725-4034, john.bravman@forsythe.stanford.edu; James R. Lloyd, Lloyd Technology Associates Inc., Phone 508-461-0753, Fax 508-4610352, jlloyd@ma.ultranet.com; Matt A. Korhonen, Cornell Univ., Phone 607-255-5190, Fax 607-255-6575, matt@msc.cornell.edu

\section{MTRS Materials Society}

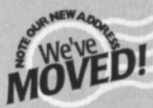
For additional meeting information contact: MRS Member Services 506 Keystone Drive,Warrendale, PA 15086 Phone: 412-779-3003, Fax: 412-779-4398 http://www.mrs.org/, E-mail: info@mrs.org 
L: Materials for High-Density Magnetic Recording

James A. Bain, Carnegie Melion Univ., Phone 412-268-3602, Fax 412-2686978, jbain@ece.cmu.edu; David Bogy, Univ. of California, Berkeley, Phone 510-642-6460 or -1339, Fax 510-642-6163, dbogy@cml.me.berkeley.edu; Tom Nolan, Komag Inc., Phone 408-576-2341, Fax 408-944-9255, tnolan@komag 1.komag.com; Kurt Rubin, IBM Almaden Research Center, Phone 408-927-2024, Fax 408-927-2100, rubinka@almaden.ibm.com

M: Integrated Magneto-Optics-Materials and Devices

Bethanie Stadler, Univ. of Minnesota, Phone 612-625-1852, Fax 612-6254583, stadler@ee.umn.edu; Miguel Levy, Columbia Univ., Phone 212-8548980, Fax 212-860-6182, miguel@cumsl.ctr.columbia.edu; Joseph Lorenzo USAF Rome Lab (EROC), Phone 617-377-2234, Fax 617-377-6765, Iorenzo@ maxwell.rl.plh.af.mil; Masud Mansuripur, Univ. of Arizona, Phone 520-621 4879, Fax 520-621-4358, masud@u.arizona.edu; Yasuyuki Okamura. Osaka/Wakayama Univ., Japan, Phone 81-6-850-6306, Fax 81-6-850-6341, okamura@ee.es.osaka-u.ac.jp; Raymond Wolfe, Lucent Technologies/Bell Labs, Phone 908-582-6672, Fax 908-582-5570,wolfe@clockwise.mh.lucent.com

\section{Ni Microelectromechanical Structures for Materials Research} Henry Guckel, Univ. of Wisconsin, Madison, Phone /Fax 608-263-4723, guckel@engr.wisc.edu; Roger Howe, Univ. of California, Berkeley, Phone 510643-7263, Fax 510-643-6637, howe@eecs.berkeley.edu; George Johnson Univ. of California, Berkeley, Phone 510-642-3371, Fax 510-642-6163, gjohnson@euler.berkeley.edu; Stuart Brown, Failure Analysis Associates, Phone 508-879-8400, Fax 508-879-9200, sbrown@fail.com; Peter Krulevitch, Lawrence Livermore National Lab, Phone 5t0-422-9195, Fax 510-422-2783, krul@IInl.gov; John Gilbert, Microcosm Technologies, Phone 617-225-0094 $x-223$, Fax 617-621-7838, jro@memcad.com

\section{O: Hybrid Materials}

Richard M. Laine Univ of Michigan, Phone 313-764-6203, Fax 313-763-4788, talsdad@umich.edu; Clément Sanchez, Université Pierre et Marie Curie, France, Phone 33-14427-5545, Fax 33-14427-4769, clems@ccr.jussieu.fr; Emmanuel Giannelis, Cornell Univ., Phone 607-255-9680, Fax 607-255-2365, emmanuel@msc.cornell.edu; C. Jeffrey Brinker, Sandia National Labs, Phone 505-272-7627, Fax 505-272-7304, jbrinker@unm.edu

\section{P: Chemical and Pyrolytic Routes to Nanostructured Powders and Their} Industrial Application

G. Beaucage, Univ. of Cincinnati, Phone 513-556-3063, Fax 513-556-3773, gbeaucag@uceng.uc.edu; Hua Duen-Wu, Millennium Inorganic Chemicals, Phone 410-354-7823, Fax 410-354-7963, bwhua@erols.com; James E. Mark Univ. of Cincinnati, Phone 513-556-9292, Fax 513-556-9239, jemark@ucbeh. san.uc.edu, http://jemcom.crs.uc.edu/; Gary Burns, Dow Corning Corp.

Phone 517-496-6090, Fax 517-496-5956, gtburns@dcrn.e-mail.com

\section{Q: Materials lssues in Chemical-Mechanical Polishing}

Jayashree Kalpathy-Cramer, LSI Logic, Phone 408-433-6528 or 503-618 4780 (Oregon), Fax 408-986-1486 or 503-618-0308 (Oregon), jaykc@lsil.com Dale L. Hetherington, Sandia National Labs, Phone 505-844-6155, Fax 505844-2991, dlhethe@sandia.gov; Michael A. Fury, mafury@aol.com

\section{R: Porous and Cellular Materials for Structural Applications}

Daniel S. Schwartz, McDonnell Douglas Corp., Phone 314-232-6835, Fax 314-232-0888, dschwartz@mdc.com; Donald S. Shih, McDonnell Douglas Corp., Phone 314-232-9202, Fax 314-232-0888, dshih@mdc.com; Haydn N.G. Wadley, Univ. of Virginia, Phone 804-982-5671, Fax 804-982-5677, haydn@ virginia.edu; Steven G. Fishman, Office of Naval Research, Phone 703-6964401, Fax 703-696-0934, fishmas@onr.navy.mil; Anthony G. Evans, Harvard Univ., Phone 617-496-0424, Fax 617-496-0601, evans@husm.harvard.edu

\section{S: Manoscale Materials Characterization Using Scanning Probes} Julia W. P. Hsu, Univ. of Virginia, Phone 804-924-7956, Fax 804-924-4576, jhsu@virginia.edu; Edward T. Yu, Univ. of California, San Diego, Phone 619 534-6619, Fax 619-534-2486, ety@ece.ucsd.edu; Clayton C. Williams, Univ. of Utah. Phone 801-585 3226. Fax 801-581-4801, clayton@mail.physics. utah.edu; Huub W.M. Salemink, IBM Zurich Research Lab, Switzerland Phone 41-1-7248416, Fax 41-1-7243170, smk@zurich.ibm.com

\section{T: Fundamentals of Nanoindentation and Nanotribology}

William W. Gerberich, Univ. of Minnesota, Phone 612-625-8548, Fax 612-6267246, wgerb@maroon.tc.umn.edu; Neville R. Moody, Sandia National Labs, Phone 510-294-2622, Fax 510-294-3410, nrmoody@sandia.gov; Shefford P. Baker, Max-Planck-Institut für Metallforschung, Germany, Phone 49-711 2095130, Fax 49-711-2095120, shefford.baker@po.uni-stuttgart.de Nancy Burnham, Ecolé Polytechnique Federale de Lausanne, Switzerland Phone 41-21-6934320, Fax 41-21-6934470, burnham@igahpse.epfl.ch

U: Electron Microscopy of Semiconducting Materials and ULSI Devices C. Hayzelden, KLA-Tencor, Phone 408-875-7479, Fax 408-571-2822, hayzelden@earthlink.net; F.M. Ross, IBM T.J. Watson Research Center, Phone 914-945-1022, Fax 914-945-2141, fmross@watson.ibm.com; C.J.D. Hetherington, Univ. of Sheffield, United Kingdom, Phone 44-114-2225345, Fax 44-114-2726391, c.j.d.hetheringtonsheffield.ac.uk

\section{Application of Synchrotron Radiation Techniques to Materials Science} Susan M. Mini, Argonne National Lab, Phone 630-252-1862, Fax 630-2527777, mini@anl.gov, or Northern Illinois Univ.; Stuart R. Stock, Georgia Inst. of Technology, Phone 757-894-6882, Fax 757-853-9140, stuart.stock@mse. gatech.edu; Dale L. Perry, Lawrence Berkeley National Lab, Phone 510-4864819, Fax 510-486-5799, dlperry@lbl.gov; Louis J. Terminello, Lawrence Livermore National Lab, Phone 510-423-7956, Fax 510-423 7040, terminello @cms1.IInl.gov

\section{W: Rapid Thermal and Integrated Processing VI}

Mehmet C. Ozturk, North Carolina State Univ., Phone 919-515-5245 Fax 919-515-3027, mco@eos.ncsu, edu; Fred Roozeboom, Philips Research abs, The Netherlands; Phone 31-40 2742767, Fax 31-40-2743352, roozeboo natlab.research.philips.com; Paul J. Timans, AG Associates, Phone 408-9352235, Fax 408-935-2785, paul.timans@agai.com; Sylvia Pas, Texas Instruments, Phone 972-995-1696, Fax 972-995-4566, s-pas@ti.com

\section{X: Frontiers of Materials Research}

John A. Emerson, Sandia National Labs, Phone 505-845-9747, Fax 505-8442894, jaemers@sandia.gov; Ronald Gibala, Univ. of Michigan, Phone 313 936-0178, Fax 313-763 4788, rgibala@umich.edu; Caroline A. Ross Massachusetts Inst. of Technology, Phone 617-258-0223, Fax 617-252-1020 caross@mit.edu; Leo J. Schowalter, Rensselaer Polytechnic Inst., Phone 518276-6435, Fax 518-276-6680, schowalt@unix.cie.rpi.edu

\section{Y. Advances in Laser Ablation of Materials}

Rajiv K. Singh, Univ. of Florida, Phone 352-392-1032, Fax 352-392-3771, rsing@mail.mse.ufl.edu; Douglas H. Lowndes, Oak Ridge National Lab, Phone 423-574-6306, Fax 423-576-3676, vdh@ornl.gov; J. Narayan, North Carolina State Univ., Phone 919-515-7874, Fax 919-515-7642, j_narayan@ncsu.edu; Douglas B. Chrisey, Naval Research Lab, Phone 202-767-4788, Fax 202-7675301, chrisey@ccsalpha2.nrl.navy.mil; T. Kawai, Osaka Univ., Japan, Phone 81 6-8798445, Fax 81-6-8752440, kawai@sanken.osaka-u.ac.jp; Eric Fogarassy, CNRS Lab PHASE BP 20, France, Phone 33-388-106257, Fax 33-388-106293, fogarasy@frcpn11in2p3.fr

\section{2: Diffusion Mechanlams in Crystalline Materials}

Yuri Mishin Virginia Polytechnic Inst. \& State Univ, Phone 540-231-9019, Fax 540-231-8919, mishin@vt.edu; Nick E.B. Cowern, Philips Research Labs, The Netherlands, Phone 31-40-2742709, Fax 31-40-2743390, cowern@natlab. research.philips.com; C.R.A. Catlow, The Royal Institution of Great Britain, United Kingdom, Phone 44-171-4092992, richard@gavy.ri.ac.uk; Diana Farkas, Virginia Polytechnic Inst. \& State Univ., Phone 540-231-4742, Fax 540-2318919 , diana@vision.mse.vt.edu; Gero Vogl, Universität Wien, Austria, Phone 43-1-313673204, Fax 43-1-3100183, vogl@pap.univie.ac.at

AA: Mechanisms and Principles of Epitaxial Crowth in Metallic Systems Luc T. Wille, Florida Atlantic Univ., Phone 561-367-3379, Fax 561-367-2662, luc@arc002.sci.fau.edu; Christopher P. Burmester, Univ. of California, Berkeley, Phone 510-486-4837, Fax 510-486-4995, chris@formulate.com; George Comsa, Universitãt Bonn, Germany, Phone 49-228-733297, Fax 49228-732551, comsa@uni-bonn.de; Ellen D. Williams, Univ. of Maryland, Phone 301-405-6156, Fax 301-314-9465, williams@surface.umd.edu; Kiyoyuki Terakura, National Inst. for Advanced Interdisciplinary Research, Japan, Phone 81-298-542704, Fax 81-298-542788, terakura@jrcat.or.jp

BB: Computational and Mathematical Models of Mlcrostructural Evolution Jeffrey W. Bullard, Univ. of Illinois, Urbana, Phone 217-244-6504, Fax 217 333-2736, jeff@puffin.mse.uiuc.edu; Marshall Stoneham, Univ. College London, United Kingdom, Phone 44-171-391-1377 or -1308, Fax 44-171-3911360, ucapams@ucl.ac.uk; Rajiv Kalia, Louisiana State Univ., Phone 504-3881112, kalia@bit.csc.Isu.edu; Long-Qing Chen, The Pennsylvania State Univ. Phone 814-863-8101, Fax 814-865-0016, chen@ems.psu.edu

\section{CC: Biomaterials Regulating Cell Function and Tlssue Development} David Mooney, Univ. of Michigan, Phone 313-763-4816, Fax 313-763-0459, mooneyd@umich.edu; Antonios G. Mikos, Rice Univ., Phone 713-285-5355, Fax 713-285 5353, mikos@rice.edu; Yoshito Ikada, Kyoto Univ., Japan, Phone 81-75-7514115, Fax 81-75-7514144, yyikada@medeng.kyoto-u.ac.jp Robert C. Thomson, W.L. Gore \& Associates, Phone 520-779-2771 x-43297 Fax 520-913-0302, rob_c_thomson@wigore.com; Kevin E. Healy, Northwestern Univ., Phone 312-503-4735, Fax 312-503-2440,kehealy@nwu.edu

\section{DD: Reliability of Photonics Materials and Structures}

Ephraim Suhir, Lucent Technologies/Bell Labs, Phone 908-582-5301, Fax 908582-5106, suhir@lucent.com; Mitsuo Fukuda, NTT Opto-electronics Labs, Japan, Phone 81-462-402837, Fax 81-462-404383, mfukuda@aecl.ntt.co.jp; Charles R. Kurkjian, Bell Communications Research, Phone 201-829-4125, Fax 201-829-5886, ckurkjia@notes.cc.bellcore.com

EF: Silicon Front-End Technology-Materials Processing and Modeling Dale Jacobson, Lucent Technologies/Bell Labs, Phone 908-582-6557, Fax 908-582-4228, dcj@bell-labs.com or dcj@lucent.com; Peter Griffin, Stanford Univ., Phone 415-725-3718, Fax 415-723-4659, griffin@gryphon.stanford.edu; Paul Packan, Intel Corp., Phone 503-613-9869, Fax 503-613-8950, ppackan@ ptdcs2.intel.com; Roger Webb, Univ. of Surrey, United Kingdom, Phone 44 1483-259830, Fax 44-1483-34139, roger.webb@ee.surrey.ac.uk; Nick Cowern, Philips Research Labs, The Netherlands, Phone 31-40-2742709, Fax 31-40 2743390, cowern@natlab.research.philips.com

\section{FF: Epitaxy and Applications of SI-Based Heterostructures}

Patricia M. Mooney, IBM T.J. Watson Research Center, Phone 914-945-3445 Fax 914-945-4581, mooney@watson.ibm.com; Derek C. Houghton, SiG Microsysterns, Canada, Phone 613-748-1334, Fax 613-748-1635, dch@ nrcphy1.phy.nrc.ca; Eugene A. Fitzgerald, Massachusetts Inst. of Technology, Phone 617-258-7461, Fax 617-258-6105, eafitz@mit.edu 


\section{EFFECTIVE AUGUST 25, 1997}

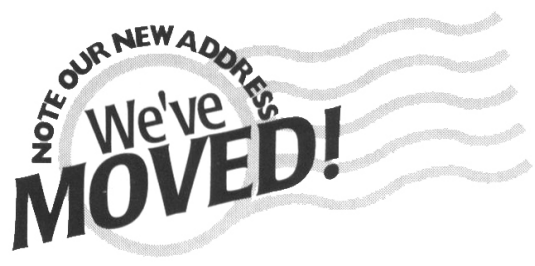

Old Address:

9800 McKnight Road, Pittsburgh, PA 15237

Please contact MRS at its new location:

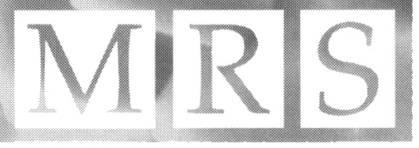

506 Keystone Drive Warrendale, PA 15086-7573 USA

Phone (operator): 412-779-3003

Phone (automated attendant): 412-779-3004

Advertising \& Exhibits: 412-779-8312

Fax: 412-779-8313

E-mail addresses and our MRS Website remain unchanged.

Information about the Materials Research Society and its full range of products and services can be found on the internet: $h$ ttp://www.mrs.org/

MRS is excited about its new headquarters facility and the opportunity it provides to advance the MRS commitment to its members and the materials research community.

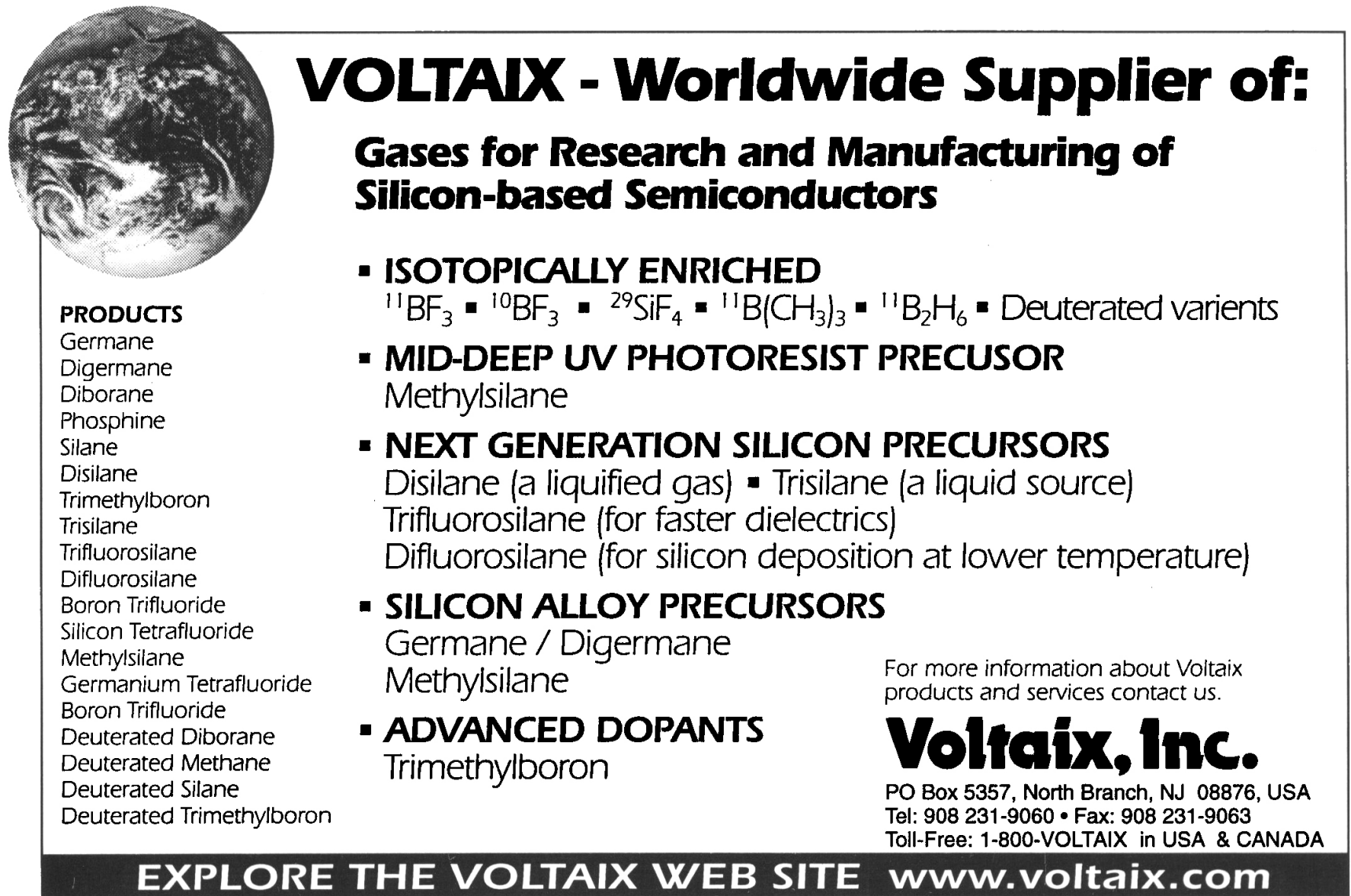




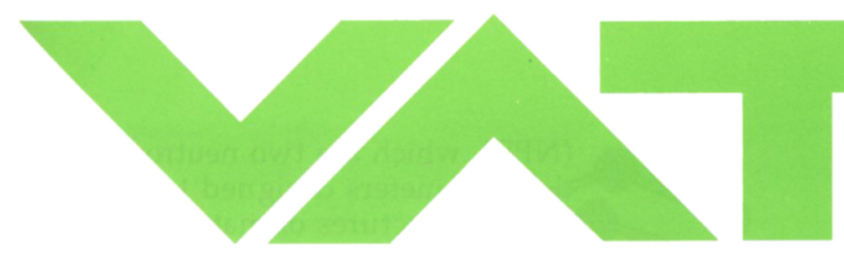

@

\section{New Catalog Vacuum Valves 2000}

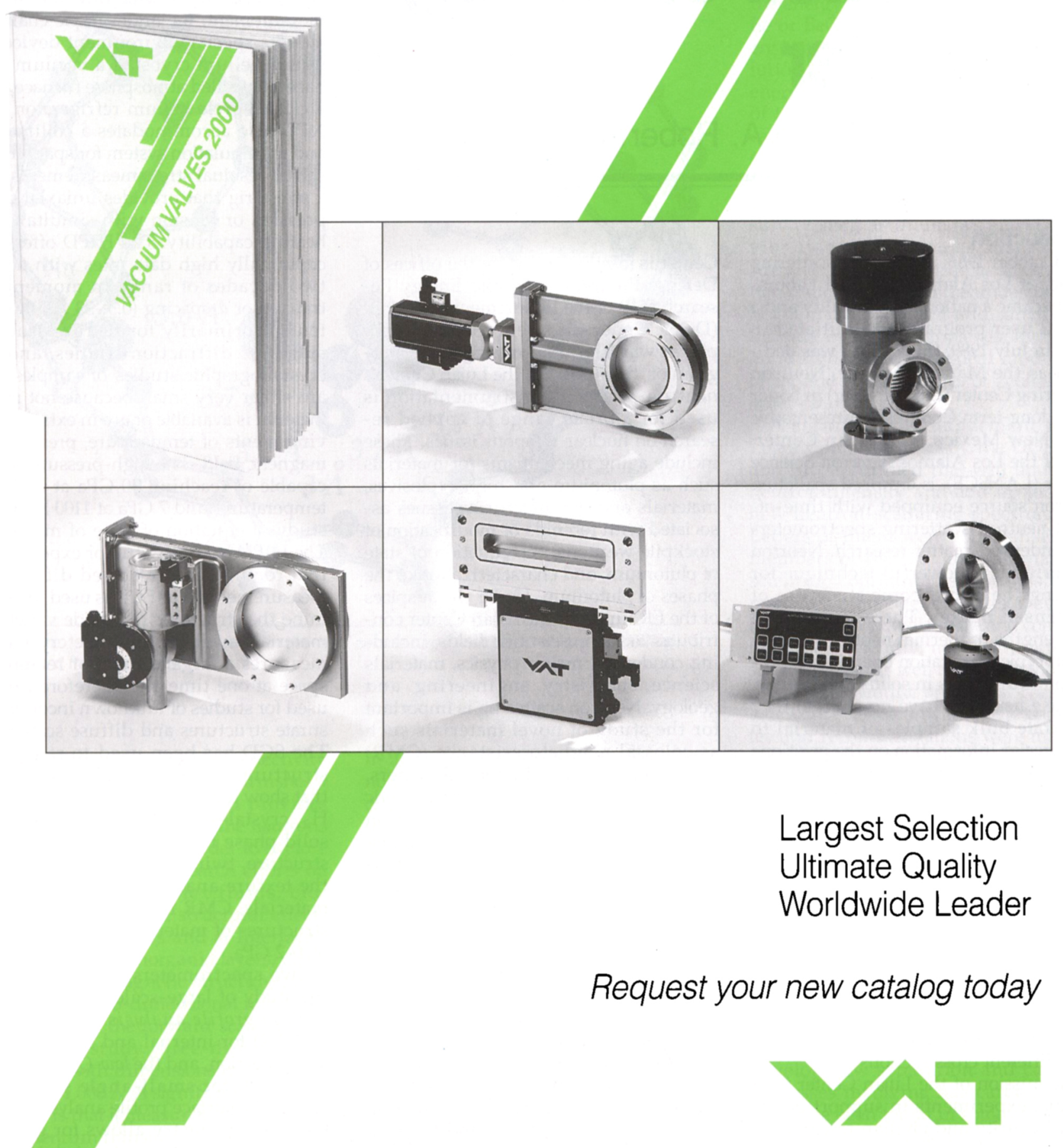

VAT, Inc., 500 West Cummings Park, Woburn, MA 01801

617-935-1446 Fax 617-935-3940 email: usa@vatvalve.com http://www.vatvalve.com 